\title{
Utility and Validity of Intracoronary Administration of Nicorandil Alone for the Measurement of Fractional Flow Reserve in Patients With Intermediate Coronary Stenosis
}

\author{
Kasumi Ishibuchi, MD; Kenichi Fujii, MD; Satoru Otsuji, MD; Shin Takiuchi, MD; \\ Katsuyuki Hasegawa, MD; Hiroto Tamaru, MD; Rui Ishii, MD; \\ Shingo Yasuda, MD; Sho Nakabayashi, MD; Wataru Yamamoto, MD; \\ Hirofumi Kusumoto, MD; Yusuke Taniguchi, MD; Mikio Kakishita, MD; \\ Yuji Shimatani, MD; Yorihiko Higashino, MD
}

\begin{abstract}
Background: Intracoronary (IC) administration of nicorandil has been proposed as an alternative choice of hyperemic agent for fractional flow reserve (FFR) measurements. This study evaluated the utility and validity of IC nicorandil administration alone to induce maximal hyperemia.

Methods and Results: Two-hundred-seven patients with coronary artery disease listed for coronary angiography with FFR were prospectively enrolled. FFR was measured after (1) IC administration of nicorandil $2 \mathrm{mg}$ (ICNIC2 $\mathrm{mg}$ ); (2) continuous intravenous (IV) adenosine triphosphatase (ATP) infusion at $150 \mu \mathrm{g} / \mathrm{kg} / \mathrm{min}$ (IVATP150); (3) IV ATP infusion at $210 \mu \mathrm{g} / \mathrm{kg} / \mathrm{min}$ (IVATP210); (4) IC administration of $0.5 \mathrm{mg}$ nicorandil during IVATP150 (ICNIC0.5 mg+IVATP150); (5) IC administration of $1 \mathrm{mg}$ nicorandil during IVATP150 (ICNIC1 mg+IVATP150); and (6) IC administration of $2 \mathrm{mg}$ nicorandil during IVATP150 (ICNIC2 mg+IVATP150). The average FFR values and the rate of achieving maximum hyperemia after ICNIC2 mg, IVATP150, IVATP210, ICNIC0.5 mg+IVATP150, ICNIC1 mg+IVATP150, and ICNIC2 mg+IVATP150 were $0.85 \pm 0.08,0.89 \pm 0.08,0.85 \pm 0.09,0.84 \pm 0.08,0.83 \pm 0.08,0.83 \pm 0.08(P<0.01)$, and $92 \%, 54 \%, 91 \%, 96 \%, 99 \%, 99 \%(P<0.01)$, respectively. The incidence of systolic aortic pressure drop, chest discomfort, and transient atrioventricular block increased in a dose-dependent manner after IV ATP infusion, but almost no adverse effects were observed after ICNIC2 mg.
\end{abstract}

Conclusions: ICNIC2 mg produced a more pronounced hyperemia than continuous IV ATP, and might be the preferred method for assessment of FFR.

Key Words: Coronary artery disease; Coronary circulation; Fractional flow reserve

$\mathbf{T}$ his study prospectively investigated the utility of intracoronary nicorandil (ICNIC) $2 \mathrm{mg}$ administration alone, compared with continuous intravenous (IV) adenosine triphosphatase (IVATP) infusion to obtain maximal hyperemia in 207 patients. The pharmacological protocol was as follows: ICNIC2 mg, IVATP of $150 \mu \mathrm{g} / \mathrm{kg} /$ min and $210 \mu \mathrm{g} / \mathrm{kg} / \mathrm{min}$, and ICNIC administration during IVATP infusion. Maximum hyperemia was achieved in $92 \%$ after ICNIC $2 \mathrm{mg}, 54 \%$ after IVATP $150 \mu \mathrm{g} / \mathrm{kg} / \mathrm{min}, 91 \%$ after IVATP $210 \mu \mathrm{g} / \mathrm{kg} / \mathrm{min}$, and $99 \%$ after ICNIC+IVATP $150 \mu \mathrm{g} / \mathrm{kg} / \mathrm{min}$. Aortic pressure drop and the incidence of chest discomfort and transient atrioventricular block increased in a dose-dependent manner during IVATP, but neither chest symptoms nor arrhythmic events were

\section{Editorial p 1986}

observed after ICNIC2 mg.

Coronary angiography (CAG), considered the referencestandard to evaluate coronary anatomy, is routinely performed, but lumen assessment correlates poorly with the physiological assessments for myocardial ischemia., ${ }^{\mathbf{1} 2}$ For functional assessment of the severity of a coronary lesion, fractional flow reserve (FFR) derived from coronary pressure wire measurements has been widely integrated in interventional practice. FFR-guided percutaneous coronary intervention (PCI) is associated with improved clinical outcomes in patients with coronary artery disease (CAD) ${ }^{36}$

Received May 10, 2019; revised manuscript received July 6, 2019; accepted July 9, 2019; J-STAGE Advance Publication released online August 14, 2019 Time for primary review: 18 days

Department of Cardiology, Higashi Takarazuka Satoh Hospital, Takarazuka (K.I., K.F., S.O., S.T., K.H., H.T., R.I., S.Y., S.N., W.Y., H.K., Y.T., M.K., Y.S., Y.H.); Department of Medicine II, Kansai Medical University, Hirakata (K.F.), Japan

University Hospital Medical Information Network 000035100

Mailing address: Kenichi Fujii, MD, PhD, Department of Medicine II, Kansai Medical University, Hirakata 573-1010, Japan. E-mail: fujiik@hirakata.kmu.ac.jp

ISSN-1346-9843 All rights are reserved to the Japanese Circulation Society. For permissions, please e-mail: cj@j-circ.or.jp 


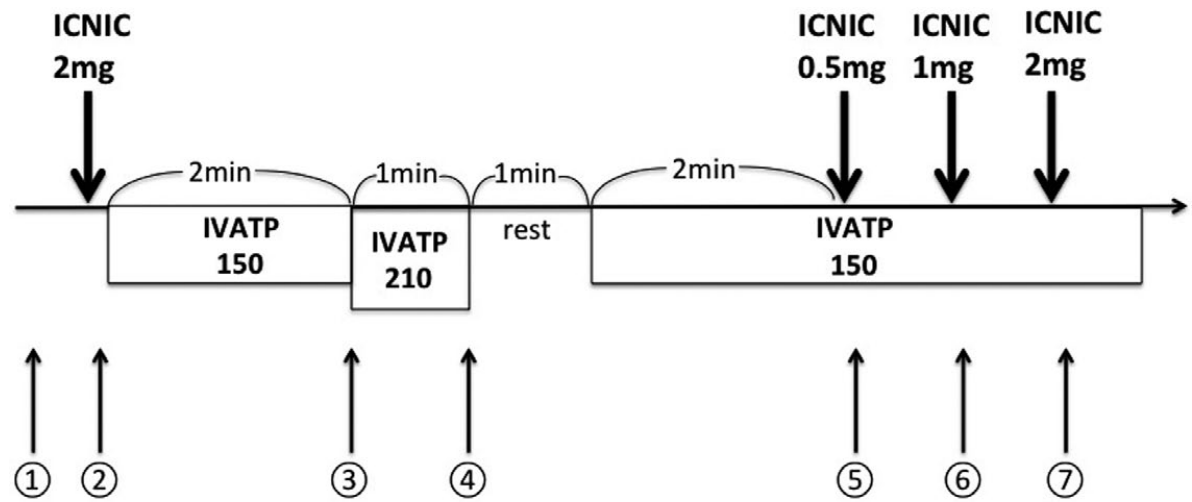

\begin{tabular}{|llll|}
\hline Record(1) & Rest & Record(5) & ICNIC0.5mg+IVATP150 \\
Record(2) & ICNIC 2mg & Record(6) & ICNIC1mg+IVATP150 \\
Record(3) & IVATP150 & Record(7) & ICNIC2mg+IVATP150 \\
Record(4) & IVATP210 & & \\
\hline
\end{tabular}

Figure 1. Study pharmacological protocol showing 7 sequential steps. ICNIC2 mg, intracoronary bolus administration of nicorandil $2 \mathrm{mg}$; IVATP150, continuous intravenous ATP infusion at $150 \mu \mathrm{g} / \mathrm{kg} / \mathrm{min}$ for $2 \mathrm{~min}$; IVATP210, continuous intravenous ATP infusion at $210 \mu \mathrm{g} / \mathrm{kg} / \mathrm{min}$ for $2 \mathrm{~min}$; ICNIC2mg+IVATP150, ICNIC2 mg during IVATP150; Pa, mean aortic pressure; Pd, mean distal coronary pressure.

For accurate measurement of FFR, the principle is to induce maximal hyperemia to minimize the effect of microvascular resistance. ${ }^{7}$ However, if coronary hyperemia is suboptimal, FFR can be erroneously high, resulting in a significant underestimation of the severity of coronary stenosis. Although IV infusion of adenosine or adenosine triphosphatase (ATP) is recommended for FFR measurements and widely used in daily clinical practice, ${ }^{\mathbf{8}, 9}$ such administration requires relatively large doses, and is associated with more systemic adverse effects and arrhythmic events. ${ }^{10}$ Recently, IC administration of nicorandil, a vasodilator that has the dual properties of a nitrate and an ATP-sensitive $\mathrm{K}^{+}$channel agonist, has been proposed as an alternative hyperemic agent to achieve maximal hyperemia. ${ }^{\mathbf{1 1 , 1 2}}$ Tanaka et al recently demonstrated that maximum hyperemia could be achieved with an IC bolus of $2 \mathrm{mg}$ nicorandil, and there was no further decrease in FFR with nicorandil doses $>2 \mathrm{mg} .{ }^{13}$ However, whether IC administration of nicorandil $2 \mathrm{mg}$ induces maximum hyperemia is unclear, so we investigated the usefulness and safety of IC bolus administration of $2 \mathrm{mg}$ nicorandil alone, compared with IV ATP infusion with additional IC nicorandil administration during the ATP infusion to achieve maximal hyperemia in patients with CAD.

\section{Methods}

\section{Selection of Patients and Study Design}

From May 2017 to October 2018, a total of 213 patients with 216 coronary artery stenoses were prospectively enrolled. Inclusion criteria for this study were moderate coronary artery stenosis defined as $50-75 \%$ stenosis based on visual estimation on angiography, age $20-85$ years, and willing to and capable of providing written informed consent. Exclusion criteria included any of the following: acute coronary syndrome within the previous month; pharmacologically treated bronchial asthma or chronic obstructive pulmonary disease; allergy to ATP or nicorandil; 2nd- or 3rd-degree atrioventricular block; severe valvular heart disease; New York Heart Association class IV heart failure; severe renal insufficiency; dissection or excessive calcification or tortuosity in the target vessel; and stenosis of a previous bypass graft. The goal of this prospectively designed study was to compare the effect of different dosages of IC nicorandil, IV administration of ATP, and the combined use of IC nicorandil and IV ATP for their potential to induce maximum and steady-state hyperemia. The primary endpoint was the difference in the rate of achieving maximum hyperemia for each vasodilatory stimulus. The major secondary endpoint was the incidence of adverse effects associated with each vasodilatory stimulus. The Ethics Committee at Higashi Takarazuka Satoh Hospital (Takarazuka, Hyogo, Japan) approved the protocols, and written informed consent was given by all patients. This study was registered as UMIN000035100 at the University Hospital Medical Information Network Clinical Trials Registry (http://www.umin.ac.jp/ctr/), which is a public trial registry approved by the International Committee of Medical Journal Editors.

\section{Catheterization}

CAG with 5- or 6-F diagnostic catheters without side holes was performed through the radial or femoral artery, in standard multiple views by experienced operators at least $30 \mathrm{~s}$ after administration of $0.1-0.3 \mathrm{mg}$ of IC nitroglycerin. After diagnostic catheterization, a 5,000-6,000 IU bolus of heparin was administered to all patients before the procedure. Next, a 0.014-inch pressure guide wire (PressureWire Verrata, Volcano, San Diego, CA, USA; PressureWire Aeris, Abbott Vascular, Santa Clara, CA, USA; or 


\begin{tabular}{lc|}
\hline \multicolumn{2}{l}{ Table 1. Baseline Patient and Lesion Characteristics } \\
Age (years) & $74(67,79)$ \\
Male & $147(71)$ \\
Body mass index $\left(\mathrm{kg} / \mathrm{m}^{2}\right)$ & $25 \pm 3$ \\
Hypertension & $148(72)$ \\
Dyslipidemia & $133(64)$ \\
Diabetes mellitus & $72(35)$ \\
Current smoker & $21(10)$ \\
Past smoker & $35(17)$ \\
Previous myocardial infarction & $57(28)$ \\
Hemodialysis & $2(1)$ \\
Atrial fibrillation & $10(5)$ \\
Clinical presentation & \\
Asymptomatic & $63(30)$ \\
Stable angina & $144(70)$ \\
Target vessel & \\
Left main & $3(1)$ \\
Left anterior descending & $135(64)$ \\
Left circumflex & $34(16)$ \\
Right coronary & $38(18)$ \\
Lesion location & \\
Proximal & $72(34)$ \\
Middle & $125(60)$ \\
Distal & $13(6)$ \\
Bifurcation & $65(31)$ \\
Quantitative coronary angiography & \\
Reference diameter (mm) & $2.7(2.3,3.1)$ \\
Minimum lumen diameter (mm) & $1.6 \pm 0.4$ \\
Diameter stenosis $(\%)$ & $40 \pm 13$ \\
\hline
\end{tabular}

Data are mean $\pm \mathrm{SD}, \mathrm{n}(\%)$, or median (interquartile range).

Optowire, Opsens Inc., Quebec City, Canada) was externally calibrated and introduced into the diagnostic catheter. The pressure transducer was advanced just beyond the tip of the guiding catheter, and it was verified that the measured pressures in the pressure wire and the guiding catheter were equal after flushing the diagnostic catheter with saline, to avoid pressure damping caused by contrast medium viscosity. The pressure wire was advanced distal to the stenotic lesion, and the Y-connector was tightened to avoid dislocation of the pressure wire. Flushing of the diagnostic catheter with saline was repeated to avoid pressure damping caused by contrast medium and blood viscosity within the diagnostic catheter. The mean distal coronary pressure $(\mathrm{Pd})$ and the mean aortic pressure $(\mathrm{Pa})$ were measured at baseline and during hyperemia induced by each hyperemic stimulus. FFR was calculated as the ratio of $\mathrm{Pd}$ to $\mathrm{Pa}$ during hyperemia and reported as an average of 5 cardiac cycles. Additionally, heart rate, aortic pressure, distal coronary pressure, ECG changes, and symptoms (chest pain, dyspnea, or headache) were continuously monitored and recorded at baseline and during hyperemia.

\section{Pharmacological Protocol}

The pharmacological protocol comprised 7 sequential steps as outlined (Figure 1). The venous line and the diagnostic catheter were adequately flushed with saline between FFR measurements with each hyperemic agent. (1) Resting Pd/ $\mathrm{Pa}$ : measured for at least $1 \mathrm{~min}$ for a stable assessment of resting physiology for the calculation. (2) IC bolus of nicorandil $2 \mathrm{mg}$ (ICNIC2 $\mathrm{mg}$ ): $10 \mathrm{~mL}$ of nicorandil ( $2 \mathrm{mg}$ in saline) injected over $5 \mathrm{~s}$, followed by $0.9 \%$ saline flush over a $5 \mathrm{~s}$ period. (3) IV infusion of standard dose ATP $(150 \mu \mathrm{g} /$ $\mathrm{kg} / \mathrm{min}$ ) (IVATP150): continuous IV ATP infusion at $150 \mu \mathrm{g} / \mathrm{kg} / \mathrm{min}$ through a large forearm vein for at least $2 \mathrm{~min}$. (4) IV infusion of high dose ATP $(210 \mu \mathrm{g} / \mathrm{kg} / \mathrm{min})$ (IVATP210): continuous IV ATP infusion at $210 \mu \mathrm{g} / \mathrm{kg} / \mathrm{min}$ through a large forearm vein for at least $2 \mathrm{~min}$. (5) IC bolus of $0.5 \mathrm{mg}$ nicorandil during continuous IV ATP infusion at $150 \mu \mathrm{g} / \mathrm{kg} / \mathrm{min}$ (ICNIC0.5 mg+IVATP150): $10 \mathrm{~mL}$ of nicorandil $(0.5 \mathrm{mg}$ in saline) injected over $5 \mathrm{~s}, 2 \mathrm{~min}$ after IV administration of standard dose ATP (150 $\mu \mathrm{g} / \mathrm{kg} / \mathrm{min})$. (6) IC bolus of $1 \mathrm{mg}$ nicorandil during continuous IV ATP infusion at $150 \mu \mathrm{g} / \mathrm{kg} / \mathrm{min}$ (ICNIC1 mg+IVATP150): $10 \mathrm{~mL}$ of nicorandil (1 mg in saline) injected over $5 \mathrm{~s}, 2 \mathrm{~min}$ after IV administration of standard dose of ATP $(150 \mu \mathrm{g} / \mathrm{kg} / \mathrm{min})$. (7) IC bolus of $2 \mathrm{mg}$ nicorandil during IV continuous ATP infusion at $150 \mu \mathrm{g} / \mathrm{kg} / \mathrm{min}$ (ICNIC2 $\mathrm{mg}+$ IVATP150): $10 \mathrm{~mL}$ of nicorandil ( $2 \mathrm{mg}$ in saline) injected over $5 \mathrm{~s}, 2 \mathrm{~min}$ after IV administration of standard dose of ATP $(150 \mu \mathrm{g} / \mathrm{kg} / \mathrm{min})$. The lowest $\mathrm{Pd} / \mathrm{Pa}$ value among the various hyperemic stimuli was considered to indicate maximum hyperemia. ${ }^{\mathbf{1 4 , 1 5}}$

\section{Quantitative CAG}

A 5- or 6-F diagnostic catheter was used to selectively cannulate the ostium of the target coronary artery. All CAGs were performed after IC administration of $0.2 \mathrm{mg}$ nitroglycerin to eliminate the effect of coronary arterial spasm. All angiographic analyses were performed by an independent investigator who was blinded to the patients' characteristics and the FFR results. The minimum lumen diameter, reference diameter, percentage diameter stenosis, and lesion length with the least foreshortening view were measured using a validated automated edge detection algorithm (CAAS 5.11, Pie Medical Imaging, Eindhoven, The Netherlands).

\section{Statistical Analysis}

Continuous variables are expressed as mean \pm standard deviation if normally distributed or as median (interquartile range) if non-normally distributed. Categorical variables are presented as numbers and percentages. The one-way analysis of variance (ANOVA) was used to compare continuous variables. Additionally, post-hoc analysis was performed for evaluation of significance. The chi-square test and Fisher's exact tests were used to analyze categorical variables. The relationship between $\mathrm{Pd} / \mathrm{Pa}$ after each hyperemic stimulus was quantified with a coefficient of determination $\left(\mathrm{r}^{2}\right)$. Agreement between $\mathrm{Pd} / \mathrm{Pa}$ after each hyperemic stimulus was assessed by Bland-Altman plots and 95\% limits of agreement. All statistical analyses were performed using JMP statistical software for Windows version 8.0.2 (SAS Institute Inc., Cary, NC, USA). A value of $\mathrm{P}<0.05$ was considered statistically significant.

\section{Results}

Of 216 lesions, 6 were excluded from the analysis because of poor quality of the pressure wave, or inability to continue with the study protocol because of unstable hemodynamics that precluded the administration of ATP. Finally, a total of 210 lesions from 207 patients were included in this analysis: 135 left anterior descending, 34 left circumflex, and 38 right coronary arteries. Baseline patient and lesion 


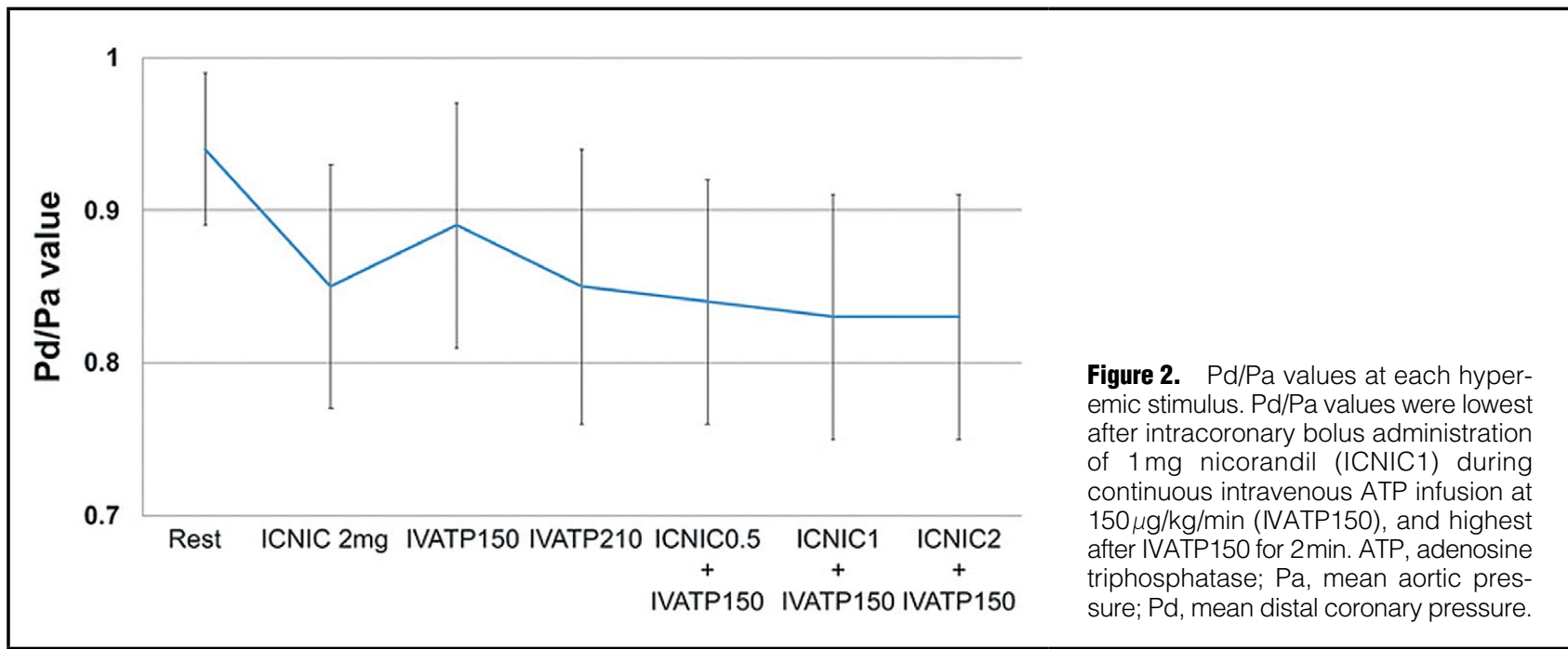

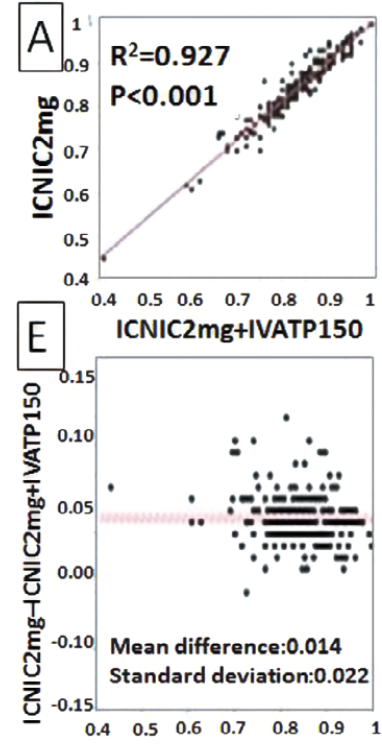

(ICNIC2mg+ICNIC2mg+IVATP150)/2
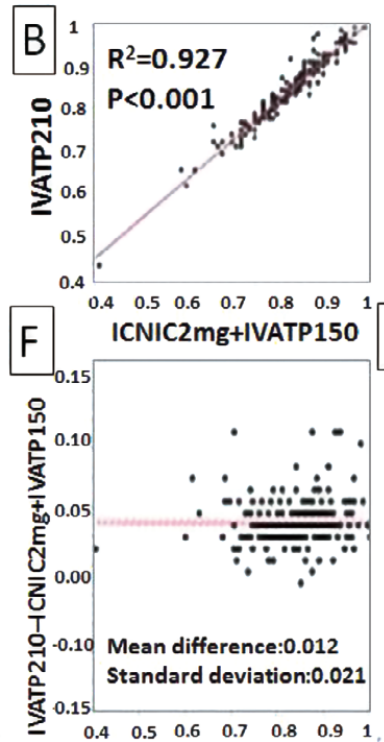

(IVATP210+ICNIC2mg+IVATP150)/2

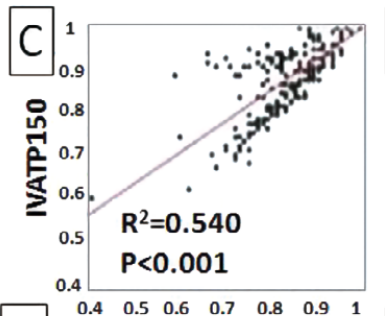

ICNIC2mg+IVATP150



(IVATP150+ICNIC2mg+IVATP150)/2
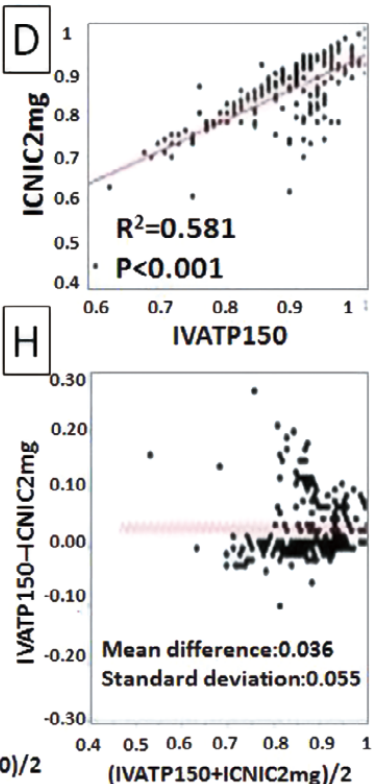

Figure 3. Correlation between each hyperemic stimulus and Bland-Altman analysis. (A-C) Strong and linear correlation between the Pd/Pa values after ICNIC2 mg+IVATP150 and after ICNIC2 mg and after IVATP210, but weak after ICNIC2 mg+IVATP150 and after IVATP150. (D) Weak correlation after ICNIC2 mg and after IVATP150. (E-H) Bland-Altman analysis for each hyperemic stimulus. Most of the Pd/Pa discrepancies were observed after IVATP150. ICNIC2 mg, intracoronary bolus administration of nicorandil 2mg; IVATP150, continuous intravenous ATP infusion at $150 \mu \mathrm{g} / \mathrm{kg} / \mathrm{min}$ for $2 \mathrm{~min}$; IVATP210, continuous intravenous ATP infusion at $210 \mathrm{\mu g} / \mathrm{kg} / \mathrm{min}$ for $2 \mathrm{~min}$; ICNIC2 mg+IVATP150, ICNIC2 mg during IVATP150; Pa, mean aortic pressure; Pd, mean distal coronary pressure.

characteristics are summarized in Table $\mathbf{1 .}$

\section{$\mathrm{Pd} / \mathrm{Pa}$ Value During Each Hyperemic Stimulus}

The average $\mathrm{Pd} / \mathrm{Pa}$ values were $0.94 \pm 0.05$ at rest, $0.85 \pm 0.08$ after ICNIC $2 \mathrm{mg}, 0.89 \pm 0.08$ after IVATP150, $0.85 \pm 0.09$ after IVATP210, 0.84 \pm 0.08 after ICNIC0.5 mg+IVATP150, $0.83 \pm 0.08$ after ICNIC1 $\mathrm{mg}+\mathrm{IVATP} 150$, and $0.83 \pm 0.08$ after ICNIC2 mg+IVATP150 (Figure 2). The value after IVATP150 was significantly higher than those after the other hyperemic stimuli $(\mathrm{P}<0.001)$. Maximum hyperemia was achieved $92 \%$ after ICNIC $2 \mathrm{mg}, 54 \%$ after IVATP 150 , 91\% after IVATP210, 96\% after ICNIC0.5 mg+IVATP 150 , $99 \%$ after ICNIC1 mg+IVATP150, and 99\% after ICNIC2 mg+IVATP150 ( $\mathrm{P}<0.001)$. The $\mathrm{Pd} / \mathrm{Pa}$ values after ICNIC $2 \mathrm{mg}$ and after IVATP210 strongly correlated with those after ICNIC2mg+IVATP150 $\left(\mathrm{r}^{2}=0.93, \mathrm{P}<0.001\right.$, and $r^{2}=0.93, P<0.001$, respectively, Figure 3). On the other hand, the relationship was relatively weak between the $\mathrm{Pd} / \mathrm{Pa}$ value after IVATP150 and that after ICNIC2 mg+IVATP150 $\left(\mathrm{r}^{2}=0.54, \mathrm{P}<0.001\right) . \mathrm{The} \mathrm{Pd} / \mathrm{Pa}$ 


\begin{tabular}{|c|c|c|c|c|c|c|c|}
\hline & ICNIC2 mg & IVATP150 & IVATP210 & $\begin{array}{l}\text { ICNIC0.5 mg+ } \\
\text { IVATP150 }\end{array}$ & $\begin{array}{l}\text { ICNIC1 mg+ } \\
\text { IVATP150 }\end{array}$ & $\begin{array}{l}\text { ICNIC2 mg+ } \\
\text { IVATP150 }\end{array}$ & $P$ value \\
\hline $\begin{array}{l}\text { Systolic Pa drop } \\
(\mathrm{mmHg})\end{array}$ & $\begin{array}{c}-9.5 \\
(-15,-3)\end{array}$ & $\begin{array}{c}-10.5 \\
(-22,-1)\end{array}$ & $\begin{array}{c}-23 \\
(-35,-13)\end{array}$ & $\begin{array}{c}-17 \\
(-28,-8)\end{array}$ & $\begin{array}{c}-22 \\
(-33,-11)\end{array}$ & $\begin{array}{c}-27 \\
(-39,-16)\end{array}$ & $<0.001$ \\
\hline $\begin{array}{l}\text { Diastolic Pa drop } \\
\text { (mmHg) }\end{array}$ & $\begin{array}{l}-4 \\
(-7,0)\end{array}$ & $\begin{array}{c}-5 \\
(-10,0.25)\end{array}$ & $\begin{array}{c}-11.5 \\
(-17,-7)\end{array}$ & $\begin{array}{c}-9 \\
(-13,-4)\end{array}$ & $\begin{array}{c}-9 \\
(-16,-5.5)\end{array}$ & $\begin{array}{c}-13 \\
(-17,-8)\end{array}$ & $<0.001$ \\
\hline $\begin{array}{l}\text { Heart rate increase } \\
\text { (beats/min) }\end{array}$ & $0(-1,2)$ & $3(0,7)$ & $7(2,11)$ & $6(2,9)$ & $6(2,10)$ & $6(2,11)$ & $<0.001$ \\
\hline $\begin{array}{l}\text { Chest } \\
\text { discomfort/dyspnea }\end{array}$ & $11(5)$ & $20(10)$ & $56(27)$ & $45(22)$ & 39 (19) & $41(20)$ & $<0.001$ \\
\hline $\begin{array}{l}\text { Transient atrioventricular } \\
\text { block }\end{array}$ & $0(0)$ & $1(1)$ & $9(4)$ & $0(0)$ & $0(0)$ & $0(0)$ & 0.002 \\
\hline $\begin{array}{l}\text { Hypotension requiring } \\
\text { any vasopressor }\end{array}$ & $0(0)$ & $0(0)$ & $1(1)$ & $2(1)$ & $2(1)$ & $2(1)$ & 0.5 \\
\hline
\end{tabular}

Data are median (interquartile range) or $\mathrm{n}(\%)$. ICNIC2 mg, intracoronary bolus administration of nicorandil $2 \mathrm{mg}$; IVATP150, continuous intravenous ATP infusion at $150 \mu \mathrm{g} / \mathrm{kg} / \mathrm{min}$ for $2 \mathrm{~min}$; IVATP210, continuous intravenous ATP infusion at $210 \mu \mathrm{g} / \mathrm{kg} / \mathrm{min}$ for $2 \mathrm{~min}$.

values after ICNIC2 $\mathrm{mg}$ at $95 \%$ limits of agreement were 0.01-0.02, whereas the $\mathrm{Pd} / \mathrm{Pa}$ values after IVATP150 at 95\% limits of agreement had a wider range (0.04-0.06) (Figure 3). Similarly, the relationship was weak between the Pd/Pa value after ICNIC2 $\mathrm{mg}$ and IVATP150 $\left(\mathrm{r}^{2}=0.58\right.$, $\mathrm{P}<0.001)$. A Bland-Altman agreement plot showed the 95\% limits of agreement between 0.03 and 0.05 (Figure 3).

\section{Hemodynamic Changes During Hyperemia}

Hemodynamic changes during the $\mathrm{Pd} / \mathrm{Pa}$ measurements and the observed adverse effect for each hyperemic stimulus are shown in Table 2. Ventricular fibrillation/tachycardia, which is a potential side effect of ICNIC, was not observed in this study. The systolic aortic pressure drop during the hyperemic stimuli was lowest with ICNIC2 mg $(-9.5(-15$, -3) $\mathrm{mmHg}$ ), and the decrease was dose-dependent during the continuous IV ATP infusion. Similarly, the heart rate during the hyperemic stimuli increased in a dose-dependent manner during continuous IV ATP infusion, but there was no increase after ICNIC $2 \mathrm{mg}$. The number of patients who experienced chest discomfort or dyspnea during the hyperemic stimuli was significantly lower after ICNIC than either IVATP150 or IVATP210 (P<0.001). Transient atrioventricular block occurred in 1 patient $(1 \%)$ after IVATP150 and in 9 patients (4\%) after IVATP210, but no arrhythmic events were observed after ICNIC $2 \mathrm{mg}$.

\section{Discussion}

The main finding of this study was that maximum hyperemia could be achieved in $92 \%$ of patients with suspected angina pectoris by administration of IC bolus of $2 \mathrm{mg}$ nicorandil. In approximately $50 \%$ of the patients, maximum hyperemic response was not achieved with IV infusion of the standard dose of $150 \mu \mathrm{g} / \mathrm{kg} / \mathrm{min}$ ATP alone. ICNIC $2 \mathrm{mg}$ was associated with significantly less chest discomfort, atrioventricular conduction disturbance, and a smaller decrease in blood pressure during the hyperemic stimulus.

FFR is considered the gold standard to measure pressure differences across a coronary stenosis to determine its severity and myocardial ischemia, based on studies demonstrating significantly better outcomes with FFR-guided PCI compared with angiography-guided PCI. ${ }^{5,16}$ However, despite strong recommendations by guidelines and increasing evidence of its cost-effectiveness, the adoption of FFR in routine clinical practice remains low, which might be linked to the requirement for hyperemic agents. Hyperemic agents such as IV adenosine can trigger transient dyspnea, chest discomfort, rhythm disturbances, and hypotension in up to $38 \%$ of cases. ${ }^{17,18}$ For these reasons, the search for cheaper, faster, and more patient-friendly methods remains relevant, and several studies have evaluated the concept of the adenosine-independent index instantaneous wave-free ratio (iFR) as an alternative method of assessing lesion severity. Another hyperemic agent, IC papaverine, was widely used to measure FFR because of its faster action of coronary vasodilation, but it has been associated with critical adverse effects such as ventricular fibrillation and polymorphic ventricular tachycardia, ${ }^{\mathbf{1 9 , 2 0}}$ so papaverine is avoided in the clinical setting. Recently, IC administration of nicorandil bolus has been demonstrated as a novel hyperemic method for invasive coronary physiological assessment. ${ }^{21,22}$ Tanaka et al demonstrated that maximum hyperemia could be achieved with an IC bolus of $2 \mathrm{mg}$ of nicorandil, and there was no further decrease in FFR with nicorandil doses $>2 \mathrm{mg} .{ }^{13}$ In accordance with the previous studies, the results of our study also showed that maximum hyperemia was achieved in $92 \%$ of the patients after ICNIC2 mg with no evidence of dyspnea, chest discomfort, or atrioventricular conduction disturbances, and a smaller decrease in blood pressure during the hyperemic stimulus.

Another important finding of this study was that maximum hyperemia was obtained in only $54 \%$ of cases by continuous IVATP infusion at $150 \mu \mathrm{g} / \mathrm{kg} / \mathrm{min}$ for $2 \mathrm{~min}$. One potential reason for this could be that IVATP150 was administered via a peripheral IV line in this study. However, IV ATP infusion was performed following the same protocol as in the previous clinical studies. ${ }^{16-18,23}$ Continuous IV infusion of ATP through a vein in the forearm is the standard method for achieving maximal hyperemia in daily practice in the catheterization laboratory. Although the rate of successfully achieving maximum hyperemia improved up to $91 \%$, the incidence of adverse effects such as dyspnea, chest discomfort, and transient atrioventricular block also significantly increased approximately 3 -fold by increasing the dose of IV ATP infusion to $210 \mu \mathrm{g} / \mathrm{kg} / \mathrm{min}$ in this study. Although the incidence of adverse effects decreased when ICNIC was administered during continuous IV ATP infusion at $150 \mu \mathrm{g} / \mathrm{kg} / \mathrm{min}, 20 \%$ of the patients had to endure chest discomfort for $2 \mathrm{~min}$. However, in line with 
previous studies, the time to achieving the lowest FFR was significantly reduced with ICNIC $2 \mathrm{mg}$, despite a lesser decrease in blood pressure than with continuous IV ATP infusion. ${ }^{11}$ Along with better hyperemic efficacy, ICNIC had lower incidence of chest discomfort and transient atrioventricular block, compared with continuous IV ATP infusion.

The results of our study suggested that ICNIC $2 \mathrm{mg}$ could be an alternative method of inducing maximum hyperemia for FFR measurement, especially in patients with bronchial asthma, hypotension, or atrioventricular conduction disturbance.

\section{Study Limitations}

This study has several limitations to note. First, although this was a prospective observational study, all data were obtained from a single center. Selection bias might exist regarding the selected target vessels with moderate coronary stenosis to evaluate FFR. Second, the administration order of hyperemic agents was not randomized. The efficacy of IV infusion of ATP after nicorandil administration could be enhanced because the half-life of nicorandil is relatively long. ${ }^{24}$ Third, a higher dose of nicorandil was not tested in this study, although a previous study has demonstrated that there was no further decrease in FFR measurement with ICNIC doses $>2 \mathrm{mg} .{ }^{13}$ Fourth, the assumption that central venous pressure (CVP) was $0 \mathrm{mmHg}$ could lead to an overestimation of the FFR value, especially for patients with higher CVP. However, a previous study reported that the difference between FFR values calculated without accounting for mean right atrial pressure $(\mathrm{Pd} / \mathrm{Pa})$ and those calculated with accounting for mean right atrial pressure ( $\mathrm{Pd}$ - right atrial pressure / $\mathrm{Pa}$ - right atrial pressure) was minimal even in patients with markedly increased mean right atrial pressure. ${ }^{25}$ Fifth, the lowest $\mathrm{Pd} / \mathrm{Pa}$ value, which was considered to achieve maximum hyperemia in this study, does not always indicate maximal hyperemia. Finally, FFR $<0.8$ as a cutoff value of pressure-derived physiological indices was established using IVATP, but not ICNIC. Therefore, further investigation is required to determine the optimal cutoff value for ICNIC to discriminate with high diagnostic accuracy between normal and abnormal myocardial perfusion.

\section{Conclusions}

ICNIC2 $\mathrm{mg}$ was safe and well-tolerated, and reduced the time required for FFR measurement. Furthermore, ICNIC $2 \mathrm{mg}$ induced a more pronounced hyperemia than continuous IV ATP, and might be the preferred method for assessment of FFR.

\section{Impact on Daily Practice}

Despite strong recommendations by guidelines, the adoption of FFR in routine clinical practice remains low because the use of hyperemic agents such as IV adenosine/ATP can trigger transient dyspnea, chest discomfort, rhythm disturbances, and hypotension. The results of our study suggest that ICNIC $2 \mathrm{mg}$ could be considered as an alternative method of inducing maximum hyperemia for FFR measurement.

\section{Acknowledgments}

The authors thank the staff in the catheterization laboratory at Higashi Takarazuka Satoh Hospital for their excellent assistance during the study.

\section{Funding}

None.

\section{Conflict of Interest Statement}

The authors have no conflicts of interest to declare.

\section{References}

1. White CW, Wright CB, Doty DB, Hiratza LF, Eastham CL, Harrison DG, et al. Does visual interpretation of the coronary arteriogram predict the physiologic importance of a coronary stenosis? N Engl J Med 1984; 310: 819-824.

2. Tobis J, Azarbal B, Slavin L. Assessment of intermediate severity coronary lesions in the catheterization laboratory. $\mathrm{J} \mathrm{Am} \mathrm{Coll}$ Cardiol 2007; 49: 839-848.

3. Kawase Y, Matsuo H, Akasaka T, Shiono Y, Tanaka N, Amano $\mathrm{T}$, et al. Clinical use of physiological lesion assessment using pressure guidewires: An expert consensus document of the Japanese Association of Cardiovascular Intervention and Therapeutics. Cardiovasc Interv Ther 2019; 34: 85-96.

4. De Bruyne B, Pijls NH, Kalesan B, Barbato E, Tonino PA, Piroth Z, et al. Fractional flow reserve-guided PCI versus medical therapy in stable coronary disease. $N$ Engl J Med 2012; 367: 991-1001.

5. Tonino PA, De Bruyne B, Pijls NH, Siebert U, Ikeno F, van't Veer M, et al. Fractional flow reserve versus angiography for guiding percutaneous coronary intervention. N Engl J Med 2009; 360: $213-224$.

6. Tanaka N, Nakamura M, Akasaka T, Kadota K, Uemura S, Amano $\mathrm{T}$, et al. One-year outcome of fractional flow reservebased coronary intervention in Japanese daily practice: CVITDEFER registry. Circ $J$ 2017; 81: $1301-1306$.

7. Pijls NH, van Son JA, Kirkeeide RL, De Bruyne B, Gould KL. Experimental basis of determining maximum coronary, myocardial, and collateral blood flow by pressure measurements for assessing functional stenosis severity before and after percutaneous transluminal coronary angioplasty. Circulation 1993; 87: 13541367.

8. Fihn SD, Blankenship JC, Alexander KP, Bittl JA, Byrne JG, Fletcher BJ, et al. 2014 ACC/AHA/AATS/PCNA/SCAI/STS focused update of the guideline for the diagnosis and management of patients with stable ischemic heart disease: A report of the American College of Cardiology/American Heart Association Task Force on Practice Guidelines, and the American Association for Thoracic Surgery, Preventive Cardiovascular Nurses Association, Society for Cardiovascular Angiography and Interventions, and Society of Thoracic Surgeons. J Am Coll Cardiol 2014; 64: $1929-1949$.

9. Windecker S, Kolh P, Alfonso F, Collet JP, Cremer J, Falk V, et al. 2014 ESC/EACTS guidelines on myocardial revascularization. Rev Esp Cardiol (Engl Ed) 2015; 68: 144.

10. Patel HR, Shah P, Bajaj S, Virk H, Bikkina M, Shamoon F. Intracoronary adenosine-induced ventricular arrhythmias during fractional flow reserve (FFR) measurement: Case series and literature review. Cardiovasc Interv Ther 2017; 32: 374-380.

11. Lee JM, Kato D, Oi M, Toyofuku M, Takashima H, Waseda K, et al. Safety and efficacy of intracoronary nicorandil as hyperaemic agent for invasive physiological assessment: A patient-level pooled analysis. EuroIntervention 2016; 12: e208-e215.

12. Kato D, Takashima H, Waseda K, Kurita A, Kuroda Y, Kosaka $\mathrm{T}$, et al. Feasibility and safety of intracoronary nicorandil infusion as a novel hyperemic agent for fractional flow reserve measurements. Heart Vessels 2015; 30: 477-483.

13. Tanaka N, Takahashi Y, Ishihara H, Kawakami T, Ono H. Usefulness and safety of intracoronary administration of nicorandil for evaluating fractional flow reserve in Japanese patients. Clin Cardiol 2015; 38: 20-24.

14. Seo MK, Koo BK, Kim JH, Shin DH, Yang HM, Park KW, et al. Comparison of hyperemic efficacy between central and peripheral venous adenosine infusion for fractional flow reserve measurement. Circ Cardiovasc Intv 2012; 5: 401 - 405.

15. Takami H, Sonoda S, Muraoka Y, Sanuki Y, Kashiyama K, Fukuda S, et al. Impact ofadditional intracoronary nicorandil administration during fractional flow reserve measurement with intravenous adenosine 5'-triphosphate infusion. J Cardiol 2017; 69: $119-124$.

16. Pijls NH, van Schaardenburgh P, Manoharan G, Boersma E, Bech JW, van't Veer M, et al. Percutaneous coronary intervention 
of functionally nonsignificant stenosis: 5-year follow-up of the DEFER Study. J Am Coll Cardiol 2007; 49: 2105-2111.

17. Davies JE, Sen S, Dehbi HM, Al-Lamee R, Petraco R, Nijjer SS, et al. Use of the instantaneous wave-free ratio or fractional flow reserve in PCI. N Engl J Med 2017; 376: 1824-1834.

18. Götberg M, Christiansen EH, Gudmundsdottir IJ, Sandhall L, Danielewicz M, Jakobsen L, et al. Instantaneous wave-free ratio versus fractional flow reserve to guide PCI. N Engl J Med 2017; 376: $1813-1823$.

19. Talman CL, Winniford MD, Rossen JD, Simonetti I, Kienzle MG, Marcus ML. Polymorphous ventricular tachycardia: A side effect of intracoronary papaverine. J Am Coll Cardiol 1990; 15: 275-278.

20. Vrolix M, Piessens J, De Geest H. Torsades de pointes after intracoronary papaverine. Eur Heart $J$ 1991; 12: 273-276.

21. Jang HJ, Koo BK, Lee HS, Park JB, Kim JH, Seo MK, et al. Safety and efficacy of a novel hyperaemic agent, intracoronary nicorandil, for invasive physiological assessments in the cardiac catheterization laboratory. Eur Heart J 2013; 34: 2055-2062.

22. Oi M, Toyofuku M, Matsumura Y, Motohashi Y, Takahashi K, Kawase $\mathrm{Y}$, et al. Utility of nicorandil for the measurement of coronary fractional flow reserve. Cardiovasc Interv Ther 2014; 29: $24-30$.

23. De Bruyne B, Fearon WF, Pijls NH, Barbato E, Tonino P, Piroth Z, et al. Fractional flow reserve-guided PCI for stable coronary artery disease. $N$ Engl J Med 2014; 371: 1208-1217.

24. Frydman AM, Chapelle P, Diekmann H, Bruno R, Thebault JJ, Bouthier J, et al. Pharmacokinetics of nicorandil. Am J Cardiol 1989; 63: 25J-33J.

25. Toth GG, De Bruyne B, Rusinaru D, Di Gioia G, Bartunek J, Pellicano M, et al. Impact of right atrial pressure on fractional flow reserve measurements. J Am Coll Cardiol Intv 2016; 9: $453-459$. 